(Aus der Universitäts-Kinderklinik in Wien [Vorstand: Prof. Dr. Cl. Pir quet].)

\title{
Die zahlenmäßige Beurteilung des Ernährungszustandes durch Indices.
}

\author{
Von \\ Dr. Richard Wagner, \\ Assistenten der Universitäts-Kinderklinik. \\ Mit 3 Textabbildungen. \\ (Eingegangen am 25. November 1920.)
}

Die Körperproportionen des Menschen waren schon im klassischen Altertum Gegenstand eifriger Studien. Wie aus Friedenthals 1 , zusammenfassender Arbeit über das Wachstum zu entnehmen ist, besaßen bereits die Ägypter, und zwar in ihrer Eigenschaft als ausübende Bildhauer einen gewissen Kanon der menschlichen Gestalt. Erst spät hat auch die ärztliche Wissenschaft und Anthropologie sich um die Gesetzmäßigkeiten des Körperwachstums gekümmert. Jeder Arzt, der bei Einzel- oder Massenuntersuchungen, z. B. bei der Begutachtung der Leistungsfähigkeit eines Individuums oder bei Bestimmung des Ernährungszustandes und der Proportionen von bestimmten Gruppen von Erwachsenen oder Kindern gezwungen ist, dieses Gutachten auf eine einfache Formel zu bringen und trotz der großen Schwankungsbreite in bezug auf Fettansatz, Muskelfülle, Gewicht und Iänge eine richtige, objektiv gültige Angabe zu machen, weiß, wie groß das Bedürfnis ist, ein reduziertes $\mathrm{MaB}$ zu besitzen, das mit Ausschaltung grober Fehler auf leicht erreichbarem Wege dieser Vielheit der Varianten Rechnung trägt und ihre komplizierten Beziehungen zueinander gesetzmäßig erfaßt und gegeneinander ausgleicht. Dieses allgemein naturwissenschaftliche Bedürfnis, komplizierte Beziehungen auf eine möglichst einfache Formel zu bringen, besteht bei Konstitutionsforschern, Anthropologen und Ärzten gleicherweise zu Recht, sowohl für theoretische Fragen der Rassenforschung als auch für rein praktische Erwägungen, z. B. Ausspeisungs- oder Erholungsbedürftigkeit von Schulkindern. Wenn dennoch kein einziges der vorgeschlagenen Maße, Indices, Quotienten usw. bislang sich durchsetzen oder gar internationale Geltung wie etwa das dekadische System verschaffen konnte, so dürfte die

1) Erg. d. inn. Med. u. Kinderheilk. 8, 9 und 11, 1912 und 1913. 
Ursache wohl in den vorgeschlagenen Maßen selbst gelegen sein, weshalb ich zunächst eine Diskussion der gangbarsten Indices der neueren Literatur vorausschicke.

Vorbedingung für die Brauchbarkeit eines Körperfüllenindex überhaupt ist, daß er eine durch Division gleichdimensionaler Größen entstandene unbenannte Verhältniszahl darstellt, so daß z. B. das sog. Streckengewicht von Reiche ${ }^{1}$ ) ausscheidet, weil hier eine dreidimensionale Größe, das Gewicht, durch eine eindimensionale, die Länge, dividiert wird, woraus eine Fläche resultiert. Schon Rohrer ${ }^{2}$ ) hat auf die Unzulänglichkeit dieses Quotienten hingewiesen, da „für einen mageren Erwachsenen (55 kg, $170 \mathrm{~cm}$ ) sich ein weit größerer Wert ergibt als für ein Kind $(4 \mathrm{~kg}, 50 \mathrm{~cm}$ )", das entspricht einem Verhältnis von $322 \mathrm{zu} 80$. Rejche (1. c.) hat zwar sein Streckengewicht nur zur Bestimmung des jeweiligen Nahrungsbedarfs verwendet und sieht gerade seinen Vorteil darin, $\mathrm{da} B$ es von allen Wachstumsgrößen die größte RegelmäBigkeit zeigt und ohne Unterbrechung von der Befruchtung bis zur Erreichung des maximalen Körpergewichts zunimmt. Ob es dieser Anforderung gerecht wird, darauf kann hier nicht näher eingegangen werden, wir behaupten nur mit Rohrer (l c.), daß das Streckengewicht als $\mathrm{Maß}$ der Körperfülle ungeeignet ist, ,was begreiflich ist, da bei einem Erwachsenen eine Zentimeterscheibe natürlich mehr wiegen wird als bei einem Kinde".

Auf Grund des Streckengewichts müßte man sich nämlich den Körper in einen Zylinder von gleicher Höhe, gleichem absolutem Gewicht und gleichem mittleren spezifischen Gewicht übergeführt denken, wodurch die Formel den Zahlenwert einer Scheibe von $1 \mathrm{~cm}$ Höhe ergibt.

Brauchbarer scheint schon der Index ponderalis von $\mathrm{Livi}^{3}$ ) $=100 \frac{\sqrt[3]{\text { Körpel gewicht }}}{\text { Körperlänge }}$ zu sein, da hier gleichdimensionale Größen miteinander in Beziehung gebracht sind. Er geht davon aus, daß das Gewicht auf eine lineare Größe gebracht werden müsse, wenn es mit einer anderen linearen Größe, d. i. der Länge verglichen werden soll, und vergleicht das Körpergewicht mit einem Wasservolumen vom gleichen Gewicht. Rohrer (1. c.) hat später die dritte Potenz des Livi-Index $=100 \frac{\text { Körpergewicht }}{\text { Körperlänge }^{3}}$ als Körperfüllenindex in Vorschlag gebracht, indem er den Livi-Index verwirft. Er argumentiert da-

1) Zeitschr. f. Kinderheilk. 15, 313, 1917.

2) Korrespondenzbl. d. deutsch. Gesellsch. f. Anthropologie usw. Bd. 39, 5, 1908.

${ }^{3}$ ) Antropometria S. 40-41 (Ulrico Hoepli 1900) und Antropometria militare 2, 21 (Roma 1905), zit. nach R ohrer.• 
bei, daß die Forderung von Livi, daß das Gewicht auf eine lineare Größe gebracht werden müsse, unbegründet sei und daß ebensogut die Länge in die dritte Potenz erhoben werden könnte. Ganz allgemein ist die Körperfülle direkt proportional einer Funktion des Volumens und indirekt proportional einer Funktion der Länge. An Stelle der Volumina, deren Messung zu umständlich ist, setzt Rohrer die Gewichte, von der Voraussetzung ausgehend, daß das mittlere spezifische Gewicht der zu vergleichenden Körper identisch ist. Warum Rohrer seinem Index den Vorzug vor dem Livi-Index gibt, der ja nichts anderes ist als die Kubikwurzel aus dem Rohrer-Index, also zur Gewinnung von vergleichenden Verhältniszahlen dasselbe leistet, hat eigentlich nur einen rein äußerlichen Grund, weil Rohrer meint, daß die Körperlänge im praktischen Urteil umgangen wird, indem meist nur Körper von annähernd gleicher Länge verglichen werden, deren Körperfülle nach den praktischen Vorstellungen sich wie ihre Volumina verhalten. Das wäre nun noch kein zureichender Grund, sich für den Rohrer-Index zu entscheiden, wenn er nicht noch den Vorteil gegenüber dem Livi-Index hätte, daß die relative Differenz der nach dieser Formel aus Körpergewioht und Länge berechneten Werte eine beträchtlich größere ist als bei der Livischen Formel, wodurch Kurvenbilder, die mit seiner Hilfe konstruiert sind, ein prägnanteres Äußeres bekommen.

Erwähnt seien in diesem Zusammenhang noch die eingehenden Studien von Friedenthal (l.c.) über das Wachstum, obwohl-sie mit der Indexfrage in keinem direkten Zusammenhang stehen. Friedenthal erblickt in der Gewichtskurve des Menschen in den meisten Fällen einen besseren Maßstab des Wachstums als in der Längenmessung. Die Zuwachskurve des Mensehen gleicht einer Parabel, wenn man die allerersten Stunden des Lebens unberücksichtigt läßt. Friedenthal unterscheidet einen Natur-' und Kulturtypus der Gewichtskurve; die Sonderstellung der menschlichen Gewichtskurve gegenüber allen anderen Säugetierarten beruht vor allem in der Länge des Pubertätsgewichtsanstiegs, namentlich beim Kulturtypus der Gewichtskurve. Ferner verweist Friedenthal darauf, daß das Höhenmaß des Menschen, fälschlich die Körperlänge genannt, sich aus funktionell nicht zusammengehörigen Größen zusammensetzt und für wissenschaftlich vergleichende Messungen alle Nachteile eines Summenmaßes besitzt. Sehr wichtig scheint die Anregung Friedenthals, wenn es sich darum handelt, ein Längenmaß als Vergleichsmaß verschieden großer Menschen zu wählen, die sog. vordere Rumpflänge, d. i. die Distanz Incisura sterni Symphyse, zu benutzen; denn, wie wir später ausführen werden, hat Pirq u et zuerst eine bestimmte Relation von Körpergewicht zur Rumpflänge (wenn auch nicht zur vorderen) in seinem sog. Pelidisi zu einem 
Hauptfaktor in der Begutachtung der Körperproportionen und des Ermährungszustandes erhoben:

Einen weiteren Fortschritt in der Indexfrage bedeutet die im Jahre 1913 von Pirquet ${ }^{1}$ ) angegebene ,einfache Tafel zur Bestimmung von Wachstum und Ernährungszustand bei Kindern". An der Hand der Camererschen Grundzahlen wurde gezeigt, daß das Verhältnis der Länge zum Körpergewichte sich im Verlaufe des Kindesalters sehr bedeutend verändert. Diese Veränderung beruht hauptsächlich darauf, daß die Länge der wenig voluminösen Extremitäten viel stärker zunimmt als die Länge des voluminösen Stammes und Kopfes. Ohne die Arbeit von Rohrer zu kennen, hat Pirquet dabei den reziproken Wert des von Rohrer angegebenen Index $=\frac{\text { Länge }^{3}}{\text { Gewicht }}$ aus den Werten von Camerer berechnet und kurvenmäßig verarbeitet. Er kommt zu dem Schlusse, daß innerhalb der späteren Lebensjahre die Proportionen sich mehr nach der absoluten Länge als nach dem Lebensalter richten. Aus Pirquets Tafel entnehmen wir, für Knaben und Mädchen getrennt, für ein bestimmtes Alter die sugehörige Länge und das dieser Länge adäquate Sollgewicht. Bis zur Einführung des sog. „Gelidusi“" oder, wie es jetzt international heißt, „Pelidisi“" ${ }^{2}$ ) durch Pirquet (1916) dienten uns in der Klinik die Ca mererschen Zahlen in der Bearbeitung durch Pirquet als wichtige Standardmaße bei der Beurteilung der Proportionen und des Ernährungszustandes von Kindern; wobei wir uns bewußt waren, daß die $\mathrm{Cam}$ ererschen Zahlen nicht Durchschnittswerte, sondern wünschenswerte Sollgewichte darstellten.

Von geringerer praktischer Bedeutung für unsere spezielle Fragestellung sind die im Jahre 1916 erschienenen Körpermaßstudien Pfa undlers ${ }^{3}$ ); sie dürfen aber hier nicht unerwähnt bleiben, da die Arbeit sowohl in ihrem Inhalt als in der Bearbeitung und Durchdringung des Materials zweifellos zu den klassischen Fundamenten der wissenschaftlichen Anthropometrie gehört. Ausgehend von der Frage, ob die Variation von Körperlänge (und Körpergewicht) eine reguläre Zufallsvariation ist, wird erwiesen, daß nach $P$ fa u ndlers eigenen Erhebungen die genannten Körpermaße tatsächlich der $\mathrm{G}$ a u ss schen Feblerfunktion folgen ${ }^{4}$ ). Pfa undler beschäftigt sich weiterhin mit der Verschiedenheit der Körperlänge nach sozialer Schichtung und kommt zu dem Schlusse, daß sich Anhaltspunkte dafür gewinnen lassen, daß man eher

1) Zeitschr. f. Kinderheilk. 6, 253. 1913.

a) $\mathbf{P}$ Pondus, e decies, li linear, di divisio, si sedentis altitudo.

3) Zeitschr. f. Kinderheilk. 14, 1, 1916.

4) In einer jüngst erschienenen Untersuchung von Baltz (Zeitschr. f. Kinderheilk. 24, 327. 1920) konnte gezeigt werden, daß die Gewichtsverteilung gleichlanger, gesunder, neugeborener Kinder dem $\mathrm{G}$ a u s s schen Fehlergesetz folgt, zum mindesten symmetrisch ist. 
von einer durchschnittlichen Ubermaßigkeit der Kinder der Reichen als von einer Unter ma Bigkeit der Ar menkindersprechen könne. Die Ursache der bisher verbreiteten, entgegengesetzten Anschauung erblickt Pfaundler in der Verwendung fehlerhafter Proportionsindices. Verf. vergleicht dieses einseitig beschleunigte Längenwachstum der Kinder der wohlhabenden Bevölkerung mit Wassertrieben von Treibhauspflanzen und nennt es Proteroplasie. Beim Studium der physiologischen Längenwachstumskurve des Menschen hat sich ergeben, daß sie durchaus nicht als Parabel angesprochen werden kann, hingegen daß das Konzeptionsalter der Körperlänge in dritter Potenz proportional ist (Konzeptionsalter $=$ bürgerliches Alter $+0,75$ Jahre). Bei gleichbleibender Statur und konstanter Körperdichte besteht somit Proportionalität zwischen dem Körpergewichte des Menschen in der Wachstumsperiode und seinem Konzeptionsalter.

Der wichtigste Abschnitt in Pfaundlers Studie beschäftigt sich mit dem energetischen Oberflächengesetz. Zunächst wird (in Gemeinschaft mit $O$. Kastner) eine Methode zur direkten Oberflächenbestimmung mittels eines erheblich modifizierten Deckverfahrens, sowie ein neues Verfahren zur Berechnung der Körperoberfläche aus linearen Körpermaßen angegeben. Die Bedeutung des Vierordt-Meehschen Koeffizienten als Staturindex und seine Abhängigkeit von Alter, Art, Körperform wird einer genauen Kritik unterworfen und der Schluß gezogen, daß die Körperoberfläche im Gegensatz zur Körperlänge, zum Körpergewichte usw. und zur Oberfläche geometrischer Gebilde überhaupt keine objektive, absolute Größe, sondern ein von der angewandten Methodik abhängiges, in gewissem Sinne rein konventionelles $\mathrm{Ma} B$ darstellt. Der Energieumsatz ist mit einer gewissen Annäherung proportional dem Werte $P^{\frac{2}{3}}$ oder $E=\propto P^{\mathrm{s}}$. Der Energieumsatz wird somit ganz allgemein proportional einer Fläche, die durch arithmetische Reduktion des Körpergewichtes in ein Flächenmaß erhalten wird. Hiermit erscheint das energetische Oberflächengesetz in durchaus neuem Lichte, indem es von der Vorstellung dieser Fläche als der wirklichen Körperoberfläche abstrahiert und an ihre Stelle eine andere, rechnerisch aus dem Körpergewicht jederzeit reproduzierbare, ideelle Fläche setzt. Zu beantworten bleibt nur die Frage nach der Größe $\alpha$. Diese kann aber nur für ganz bestimmte Bedingungen als Konstante angenommen werden. Nicht nur bei einzelnen Tierspezies kommen Differenzen zwischen $\frac{E}{O}$ und. $\frac{E}{P^{\frac{3}{3}}}$ um rund $100 \%$ (weiße Maus) vor, sondern auch in den einzelnen Lebensaltern zeigt der Vi e r ord t - Me e h sche Koeffizient beträchtliche Abweichungen. Pfaundler sagt in Anlehnung an Hösslins Ausführungen wörtlich: „Auch wenn man das Zahlenmaterial durchaus akzeptiert, das zur Stütze des Oberflächen- 
gesetzes bisher vorgebracht wurde, bleibt es zweifelhaft, ob sich die Proportionalität des Energieumsatzes tatsächlich auf die Körperoberfläche als solche bezieht oder aber auf die Größe $P^{\frac{z}{3}}$; in letzterem schon von anderer Seite ins Auge gefaßten Falle würden sich Deutungsmöglichkeiten jener Proportionalität ergeben, die zum Oberflächengesetz keinen Bezug mehr haben." Ich habe Pfaundlers Diskussion des energetischen Oberflächengesetzes etwas breiter dargestellt, weil Pirquet von einer ganz anderen Seite zur $2 / 3$-Potenz des zehnfachen Gewichtes gekommen ist. Er ging dabei von der Sitzhöhe (= Länge des sitzenden Rumpfes, von der Sitzfläche bis zur Scheitelhöhe) als linearem Maß aus. Sie kann in sehr einfacher Weise gemessen werden, indem man den Abstand der horizontalen Sitzunterlage von einer Fläche bestimmt, die horizontal auf den Kopf aufgelegt wird. Ein Hauptunterschied in der Bestimmung des Energieumsatzes und damit des Nahrungsbedarfs nach Pirquet aber liegt darin, daß Pirquet. nicht die Wärmeproduktion auf die Körpergewichtseinheit im Auge hat, sondern die von seiner ideellen Ernährungsfläche $(\sqrt[3]{10 \cdot \text { Gewicht } t})^{2}$ resorbierte und ausgenützte Nahrungsmenge.

Nach dieser Exkursion in das Gebiet des Energieumsatzes führt uns unser Weg nun wieder zurück zu unserem Hauptthema der Körperfüllenindices. Ich komme hiermit zu dem schon früher erwähnten Index Pirquets ${ }^{1}$ ), dem Pelidisi, jenem Index, der von uns in Wien derzeit sowohl in der Klinik als auch bei Massenuntersuchungen von Schulkindern als Körperfüllenindex seit Jahren in größtem Stil benutzt wird. Pirquet hat bei seinen Studien über Herz, Pulsfrequenz und Körpergewicht die Entdeckung gemacht, daB ein bislang sehr wenig beachtetes Körpermaß, die Sitzhöhe, in enger Beziehung zur dritten Wurzel aus dem zehnfachen Gewicht steht ${ }^{2}$ ). Pirq u ets ursprünglicher Versuch ging dahin, ein reduziertes Idealgewicht aufzustellen, das von dem akzessorischen, von äußeren Bedingungen abhängigen Faktor des individuell schwankenden Fettgehaltes abstrahieren sollte. Dieser Versuch ist nun allerdings nicht gelungen, weil das Fett nicht einfach nur das Unterhautzellgewebe, sondern auch alle inneren Organe diffus erfüllt. Der Gang der Körpergewichtskurve ist eben allein als Maß des Ennährungszustandes und der Körperproportionen nicht geeignet, sondern muß erst in Beziehung gebracht werden zu einem anderen Körpermaß. Als dieses andere Maß hat Pirquet die Sitzhöhe erkannt. Da Beinlänge und Rumpflänge ihre eigenen Wachstumsgesetze in sich

1) Zeitschr. f. Kinderheilk. 14, 211. 1916.

2) Herr Prof. Dr. Cl. Pirquet hat mir sein reiches, bisher nicht veröffentlichtes Kurvenmaterial gütigst zur Einsicht überlassen, in welchem seine anthropometrischen Studien des Wachstums überhaupt und der Beziehungen zwischen dem Wachstum des Skeletts und der Organe niedergelegt sind. 
bergen, wird die Wachstumskurve der Standhöhe als eines Summenmaßes die wahren Verhältnisse des Körperwachstums nur verwischt wiedergeben. Die Sitzhöhe leistet in dieser Hinsicht viel bessere Dienste, wenngleich wir zugeben müssen, daß sie auch ein Summenmaß ist. Nun ist aber die wahre Rumpflänge, d. i. die Distanz Incisura sterniSymphyse, für Massenuntersuchungen aus mehrfachen Gründen nicht geeignet; sie könnte erstens nur nach Entkleidung gemessen werden, die Messung ist nicht so einfach ausführbar, sie müßte mit Zirkel ausgeführt werden, der hauptsächlich störende Faktor der Beinlänge ist in beiden Fällen ausgeschaltet und im übrigen ist ja entwicklungsgeschichtlich der Kopf ein sehr wesentlicher Bestandteil des Rumpfes.

Die Sitzhöhe, d. i. also die Distanz von der Sitzfläche zur Scheitelhöhe, wird beim Kind in aufrecht sitzender Stellung gemessen, beim Säugling in liegender Stellung auf einer horizontalen Unterlage, am besten mittels der Epsteinschen Meßbank. Pirquets Zahlen über Sitzhöhe und die Beziehung derselben zum Körpergewicht stammen teils aus eigenen Messungen, und zwar bei Föten, Neugeborenen, Säuglingen und Kindern von 1-14 Jahren, teils aus den Messungen von Quetelet, Weißenberg und West $\left.{ }^{1}\right)$. Aus allen Messungen hat sich übereinstimmend ergeben, daß die Sitzhöhe fast gleich ist der dritten Wurzel aus dem zehnfachen Körpergewicht oder, anders ausgedrückt, daß ein Würfel mit der Sitzhöhe als Seitenlänge, also ein Würfel, in dem der Mensch gerade aufrecht sitzen kann, mit Wasser gefüllt, das zehnfache Gewicht haben würde. Diese Indexzahl $(\sqrt[3]{10 \cdot \text { Gewicht }}$ : Sitzhöhe) wurde von Pirquet als Pelidisi bezeichnet. Aus Pirquets Individualuntersuchungen geht hervor, daß die Schwankungen des Pelidisi in allen Altersgruppen ziemlich ähnlich sind. „Bei den Kindern erreichen dio fetten Individuen die Zahl 100, die mageren bleiben unter 90. Bei den Erwachsenen ist außer dem Fettpolster auch die Muskulatur zu berücksichtigen, welche eine Erhöhung des Index um etwa fünf Grade bewirkt. Die Mageren sind unter 95, die Fetten über 105."

Auch die Durchschnittszahlen, die /aus Messungen der einschlägigen Literatur gewonnen wurden, führten zu ähnlichen Resultaten: in der Zeit der physiologischen Magerkeit liegen sie unter 95, in der Zeit der ersten Fülle und dann wieder beim Erwachsenen liegen sie höher. Indem das Extremitätengewicht durch das Gewicht des Kopfes kompensiert wird, erhält trotz des Wachstums der Extremitäten und des entsprechend zunehmenden Gewichts derselben doch die Sitzhöhe vom Foetus bis zum Erwachsenen ihre gleiche Beziehung zum Körpergewicht. Daß bei groben Deformitäten die Sitzhöhe nicht ein-

1) Literatur vgl. Pirquet, Zeitschr. f. Kinderheilk. 14, 228. 1916. 
wandfrei zu messen und damit die Pelidisibestimmung nicht durchführbar ist, braucht wohl nicht näher ausgeführt zu werden. Hier könnte, wie Pjrquet meint, die Beziehung des Brusuumfanges zum Körpergewicht einen Ersatz bieten. Fin weiterer Finwand wäre etwa, daß das Knochengewicht individuellen Schwankungen unterworfen und da $B$ für diese Schwankungen im Pelidisi nichts vorgesehen ist. Dieser Einwand trifft aber auch alle anderen Körperfüllenindices in gleicher Weise.

Die Bestimmung des Pelidisi kann im Einzelfalle zunächst zum Vergleich des Wechsels im Ernährungsıustand bei demselben Individuum dienen. Hier hat schon jeder Grad Pelidisi Bedeutung. Ein Grad entspricht einer Veränderung um 3\% des Körpergewichts, zehn Grade einer Veränderung um 27\%, 20 Grade emner Veränderung um 49\%. Die therlegenheit der Pelidisibestimmung gegenüber der einfachen Körpergewichtsbestimmung kommt ganz besonders bei Kindern, die im Wachstum befindlich sind, zum Ausdruck Ein im.Körpergewicht gut zunehmender Säugling z. B. kann dabei dennoch in seinem Pelidisi stehenbleiben, weil die Sitzhöhe gleichzeitig wächst. Wenn hingegen bei einem Säugling neben dem allgemeinen Längenwachstum eine starke relative Vermehrung des Fettgewebes erfolgt, so wird das in seinem Pelidisi auch deutlich zu erkennen sein.

Beim Vergleich des Ernährungszustandes versehiedener Individuen kommen nur Unterschiede im Pelidisi um mehrere Grade in Betracht. Hier spielen nämlich bei gleicher Sitzhöhe Variationen in der Länge der Extremitäten, dem Umfange des Stammes und damit dem Gewicht der inneren Organe und (besonders bei Föten und Neugeborenen) Verschiedenheiten des Kopfgewichtes eine Rolle. Es können dadurch Gewichtsdifferenzen bedingt sein, die trotz eines ähnlichen Fettpolsters mehrere Grade betragen können.

Bei Massenuntersuchungen zum Zwecke statistischer Erhebungen ist es zur Bestimmung des Pelidisi nicht nötig, die Messung der Sitzhöhe und die Gewichtsbestimmung unbekleidet durchzuführen. Wenige Millimeter Stoffdicke machen für die Messung der Sitzhöhe keinen Unterschied; und bei der Gewichtsbestimmung kann leichte Kleidung durch Abzug von einem Grade Pelid isi (entsprechend $3 \%$ des Bruttogewichts) ausgeglichen werden. Bei den Massenuntersuchungen wird jetzt in der folgenden Weise vorgegangen: Es werden die Schuhe abgelegt, hingegen eine ungefähr gleich leichte Bekleidung belassen. An einigen Fällen wird nun stichprobenweise zunächst die durchschnittliche Tara für jede einzelne Schulklasse bestimmt und gleichmäßig bei allen Wägungen in Abzug gebracht. Die Berechnung des Pelidisi ist mit Hilfe eines Rechenschiebers sehr einfach zu lösen, noch leichter mit einer entsprechenden Tabelle $\mathbf{1}$ ).

1) Pirquet, System der Ernährung II. Band, S. $288 \mathrm{ff}$. 
Zusammenfassend läßt sich also sagen, daß bei gleicher Entwicklung von Muskulatur und Fettpolster das Verhältnis zwischen dem Kubus der Sitzhöhe und dem Körpergewichte in allen Lebensaltern annähernd gleich ist. Beim muskelkräftigen Erwachsenen und beim fetten Säugling ist der Kubus der Sitzhöhe gleich dem zehnfachen Körpergewicht. Das Pelidisi ist in diesen Fällen gleich 100; beim heranwachsenden Kinde ist es durchschnittlich ungefähr 94,5, bei äußerster Abmagerung kann es bis auf 78,2 herabsinken.

Außer in der Universitäts-Kinderklinik in Wien wird die Pelidisi bestimmung im großen Stil in der amerikanischen Kinderhilfsaktion in Deutschösterreich durchgeführt, und zwar sowohl zur Erhebung der Ausspeisungsbedürftigkeit als auch zu statistischen Zwecken. Kombiniert mit einer gleichzeitigen Begutachtung des Ernährungszustandes nach Blutgehalt, Fettgehalt, Turgor und Beschaffenheit der Muskulatur dient das Pelidisi als der ziffernmäßige Ausdruck der Ausspeisungsbedürftigkeit von Schulkindern. Erstrebt wird gegenwärtig im Schulalter die Ausspeisung möglichst vieler Kinder, natürlich nach Maßgabe der verfügbaren Portionen. Derzeit gilt ein Pelidisi $=94$ als Grenzwert; d. h. alle Kinder mit Pelidisi $=94$ und darunter werden ausgespeist. Ferner wurden sämtliche, in die Ausspeisung eingereihte Schulkinder Deutschösterreichs länderweise erfaßt und der Pelidisibestimmung unterworfen.

Wenn auch gegen die Pelidisibestimmung Einwände erhoben werden können, soviel steht jedenfalls fest, daß sie für eine fortlaufende Individualuntersuchung brauchbar ist, und bei Massenuntersuchungen leistet sie jedenfalls zur ersten groben Aushebung aus einem großen Material die besten Dienste. Der ärztliche Blick wird in jenen Ausnahmefällen (Hydrocephalus, Rachitis, Wachstumsstörungen) sinngemäB korrigierend und nivellierend eingreifen, wo die $\mathrm{Zahl}$ allein versagt ${ }^{1}$ ).

Wir wollen nun noch weiter Umschau halten in der einschlägigen Literatur und hervorheben, was für unsere Frage von Wichtigkeit ist. Aus dem Jahre 1916 liegen von nicht ärztlicher Seite, und zwar von Guttmann $n^{1}$ ) exakte Daten über individuelle körperliche Entwicklung vor. Wenngleich auch dabei die erhobenen Meßresultate in keine Beziehung zueinander gebracht wurden, so liegen hier dennoch verläßliche Messungen von Größe, Brustumfang und Gewichtsbestimmungen vor; die Individuen sind stets am Geburtstage gemessen und dieselben Individuen sind durch eine Reihe von Jahren, viele durch $28 \mathrm{Jahre}$ hindurch beobachtet worden. Immerhin sind hier liebe- und wertvolle Einzelbeobachtungen zusammengetragen, wertvoll für den, der kritisch

1) Einige Beispiele individueller körperlicher Entwicklung. Zeitschr. f. Kinderheilk. 13, 248. 1916. 
ein solches Material zu sichten und zur Aufstellung von Regeln zu verarbeiten versteht.

Kurz erwähnt seien hier noch die Untersuchungen von $R e i c h \mathrm{e}^{1}$ ) über das Wachstum der Frühgeburten in den ersten Lebensmonaten. Reiche findet, daB das Wachstum der Frühgeburten nach denselben Wachstumsregeln erfolgt, die für die entsprechenden Monate nach der Refruchtung gelten. In einer zweiten und dritten Mitteilung behandelt Reiche das Wachstum des Brust- und Kopfumfanges, sowie das Wachstum der Zwillingskinder. Die Lebensfähigkeit einer Frühgeburt ist an ein gewisses MinimalmaB des Brustumfanges gebunden. Die Wachstumskurve des Kopfumfanges geht in den letzten Fötalmonaten der Brustumfangskurve fast parallel und flacht sich erst in den ersten Monaten nach der rechtzeitig erfolgten Geburt ab.

Aus dem Jahre 1917 liegt eine Untersuchung von Schlesinger ${ }^{2}$ ) vor über das Wachstum der Knaben und Jünglinge vom 6 . bis 20 . Lebensjahre. Das Material, das von 10000 Knaben und Jünglingen stammt und Knaben aus allen Bevölkerungsschichten umfaßt, ist nach dem Entwicklungszustand und der Konstitution des einzelnen Kindes und nach dem sozialen Milieu gruppiert. In sieben Tafeln ist am Ende der Arbeit das Zahlenmaterial niedergelegt. Sie enthalten eine Zusammenstellung von Größe und Gewicht, sowie von Größe, Gewicht und Brustumfang in den einzelnen Jahren nach verschiedenem Milieu und Entwicklungszustand. Diese Tafeln können als Ergänzung der oben erwähnten Pirquet-Camererschen Tabelle dienen, nach deren Muster sie angefertigt sind. In der Indexfrage hält Schlesinger in thereinstimmung mit der wissenschaftlichen Kritik von Pfaundlers nur den Livischen Index ponderalis $\frac{100 \sqrt[3]{P}}{L}$ oder den dem Rohrerindex reziproken Index Pirquets $\frac{L^{3}}{P}$, sowie das Pirquetsche Pelidisi für brauchbar. Hingegen wird das Pignet'sche Verfahren, die Bestimmung des coéfficient de robusticité $x=h-\langle b+k)$ (h= Länge, $b=$ Brustumfang, $k=$ Gewicht) verworfen.

Am Schlusse möchte ich nochmals etwas näher auf den sog. Ruohrer index $\frac{\text { Körpergewicht } \cdot 100}{\cdot \text { Länge }^{3}}$ eingehen, der im laufenden Jahre in Berlin scheinbar ausgedehntere praktische Anwendung gefunden hat. Ein Hinweis auf seine praktische Anwendung findet sich in einer Arbeit aus der Krausschen Klinik , Uber die Beriehung des proportionellen Brustumfanges zum Index der Körperfülle bei männlichen Individuen im Wachstumsalter" von Max Berliner $\left.{ }^{3}\right)$. Es wird darauf hinge-

1) Zeitschr. f. Kinderheilk. 12, 369. 1915; 13, 332 und 349. 1916.

2) Zeitschr. f. Kinderheilk. 16, 265.1917.

3) Berl. klin. Wochenschr. 1920, Nr. 2, S. 33. 
wiesen, daß „Brugsch in seiner Lehre von der ärztlichen Beurteilung des gesunden und kranken Menschen die Ergebnisse seiner Untersuchung über das Verhältnis des Brustumfanges zur Körperlänge, des sog. proportionellen Brustumfanges, an einem großen Material zusammenestellt und auf die hohe Bedeutung der Größe des proportionellen Brustumfanges für die Konstitution des Menschen hingewiesen hat". Berliner hat nun - allerdings nur an einem kleinen Material (60) -

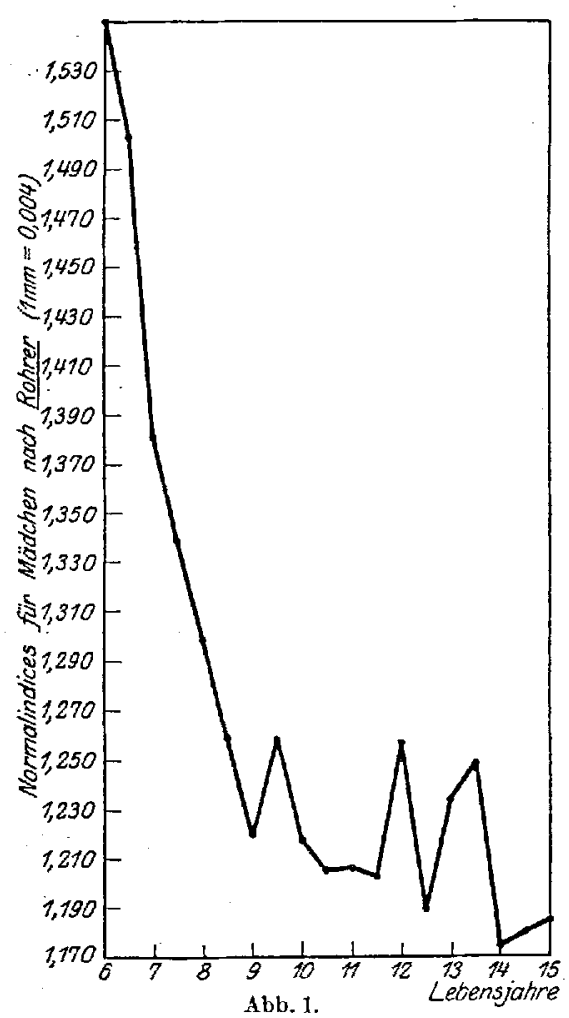

bei einer Anzahl von Knaben und Jünglingen zwischen dem 10 . und 18. Lebensjahre systematisch die Körperlänge, das Gewicht und den proportionellen Brustumfang festgestellt und in Relation zum Index der Körperfülle gebracht. Unabhängig vom Alter ergibt sich bei zunehmendem Brustumfang ein deutliches. Wachsen des Index der Körperfülle. Auch beim Vergleich mit dem Längenwachstum ergibt sich, daß die Schwankungen der Durchschnittswerte für den Rohrerindex und den proportionellen Brustumfang miteinander vollkommen parallel gehen. Trotz gleicher Körperlänge sind der proportionelle Brustumfang und der Index der Körperfülle, untereinander wieder annähernd parallel, so regellos im Vergleich zum Längenwachstum, daß auch hier von einem Abhängigkeitsverhältnis nicht die Rede sein kann. Die Zahl der Messungen scheint mir zu gering, als da $B$ daraus weitgehende Schlüsse gezogen werden könnten. Standardzahlen aus 60 Messungen sind jedenfalls nieht zu gewinnen. Darauf erhebt auch die Arbeit keinen Anspruch. Anders steht jedoch die Sache, wenn mit Hilfe der Roh rerschen Verhältniszahl ein Normalindex angegeben wird, auf den Einzel- und Massenuntersuchungen zur Begutachtung des Ernährungszustandes zu beziehen sind.

Im Buchhandel (bei $\mathbf{J}$ ulius S pringer, Berlin) ist nun in der jüngsten Zeit eine Tafel zur Bestimmung des Emährungszustandes des Schulkindes erschienen, herausgegeben vom ürztlichen Beirat der Kinder- 
hilfsmission der Religiösen Gesellschaft der Freunde (Quäker) von Amerika (Czerny, Gottstein, Rost, Rott, Rubner, Tugendreich). ${ }^{1)}$ Die Tafel enthält in übersichtlicher Form die zu den einzelnen Gewichten in Kilogramm und Körperlängen in Zentimeter zugehörigen Rohrerindices. Die Wertung des auf dieser Haupttafel abgelesenen Index erfolgt durch Beziehung auf den Normalindex der entsprechenden Aitersklasse. Der zu jeder Altersklasse gehörende, als Normalindex angenommene Wert ist aus beigefügten thersichtstabellen zu ermitteln, welche für Knaben und Mädchen von 6-15 Jahren getrennt angelegt sind. Auf diesen Úbersichten läßt sich die prozentuale Abweichung vom Normalindex von 5 zu $5 \%$ ablesen. Die Normalwerte sind als kennzeichnend für deutsehe Kinder angenommen worden. Hauptbedingung für die Verwertbarkeit einer solchen Tafel ist, daß die „Normalwerte" wirkliche Normalwerte sind. Aus wie vielen Messungen sie gewonnen sind, ist nicht angegeben. Es verlohnt daher der Mühe, die Normalindices, die Längen und Gewichte etwas näher ins Auge zu fassen und auf ihre Brauchbarkeit zu prüfen. Wenn wir, z. B. für Mädchen, die Normalindices graphisch darstellen, so genügt schon ein Blick auf diese Zeichnung, um zu erkennen, da $ß$ sie nicht entsprechend ausgeglichen ist (vgl. Abb. 1); noch
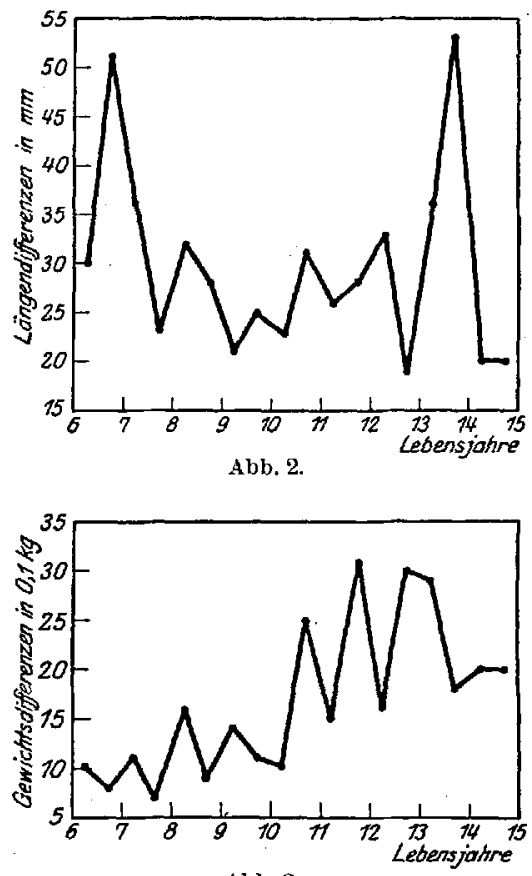

Abb. 8 . deutlicher werden diese Verhältnisse, wenn man wie in Abb. 2 und 3 nur die Differenzen der aufeinander folgenden Längen und Gewicht in einer Kurve verarbeitet. Es zeigt sich, daß die Kurve für Mädchen fünf Tiefpunkte. enthält, der tiefste bei Mädchen von 14 Jahren, und die Kurve für Knaben vier. Tiefpunkte, der tiefste bei Knaben mit 10\%/2 Jahren. Eine' solche Kurve kann nicht ausgeglichen sein, sie muß vielmehr das Produkt von Zufallsbefunden darstellen, was darauf hindeutet, daß das Material, aus dem sie gewonnen wurde, nicht allzu groß war. Damit hat sie aber die Eignung zu einer Normalindexkurve verloren. Es wäre wünschenswert gewesen, die Normalindices hätten zwar weniger Dezimalen, die ja doch nur einer

1) In der zweiten Auflage sind die Herausgebernamen fortgelassen. 
50 . R. Wagner: Die zahlenmäßige Beurteilung des Ernährungszustandes usw.

Pseudogenauigkeit entsprechen, wären dafür aber so beschaffen, daß ihr Gang einer sinngemäß konstruierten Kurve entspricht. Wäre die Kurve graphisch ausgeglichen worden, so hätte sie an praktischer Brauchbarkeit nur gewonnen. Die Methode der graphischen Interpolation ist ja überall dort erlaubt oder sogar geboten, wo man mangels entsprechender Beobachtungsdaten dennoch zu richtigen $Z$ wischenwerten gelangen will. Die Technik dieser Korrektur ist allerdings nur in der Hand des mathematisch Denkenden einwandfrei. Wir halten die erwähnte Tafel in dieser Form jedenfalls nicht für geeignet, sehen keinen Vorteil gegenüber dem Pelidisi Pirquets, da die Normalindices nicht vertrauenerweckend sind, und anerkennèn höchstens ihre äußere Form wegen der Kontrastwirkung von Rot und Schwarz (die Normalindices sind nämlich mit roter Farbe geschrieben).

Damit ist nun nichts gegen den Rohrerindex gesagt, bei entsprechender Korrektur der Tafel könnte auch er ganz brauchbar werden; nur ist nicht einzusehen, warum das Pelidisi, von dem schon nachgewiesen ist, daß es für alle Lebensalter in Normalfällen ungefähr gleich 100 ist, nicht dem Rohrerindex vorzuziehen wäre, wenn er in der Wachstumsperiode solche Schwankungen durchmacht. Vielleicht ist die Erklärung dafür jene Stimmung, aus der heraus von einigen Autoren in Berlin auch das Nemsystem verworfen wurde, ehe es theoretisch und praktisch geprüft worden war.

Nach Abschluß des Manuskripts erschien in der Münchner med. Wochenschrift 1920, Nr. 45, S. 1296 eine kurze Abhandlung von Dacha uer und Lampart. Diese Autoren haben an über 18000 Kindern den Rohrerschen Index erprobt und kennzeichnen den Erfolg als einen sehr schlechten. Zwischen der Begutachtung des Ernährungszustandes durch einfachen Inspektionsbefund und durch zahlenmäBigen Ausdruck mittels des Rohrer-Index bestehen krasse Differenzen Der Ansicht der Verfasser, daß die Unterernährung eines Kindes überhaupt durch keinen Index festgestellt werden kann, sondern daß dazu neben den anamnestischen Erhebungen sorgfältige Ernährungs- und Stoffwechselversuche notwendig seien, können wir uns hingegen nicht anschließen; denn der Zweck eines brauchbaren Ernährungsindex scheint uns ja eben die Ersetzung komplizierter Methoden durch eine einfache zu sein.

Ferner weist Oeder in Nr. 47, S. 1368 der Münch. med. Wochenschr. 1920 darauf hin, daß der Roh r e r sche Index nichts anderes sei als der alte $B$ uf fon sche Index $\frac{P}{L^{3}}$, der schon vor mehreren Menschenaltern (auch von $Q$ uetelet) als falsch erkannt worden war.

Wien IX/2, Lazarettgasse 14. 